\title{
La transformation de l'éducation au fil du temps, une longue marche
}

Introduction

Introduction. The transformation of education over time: A long march

Introducción. La transformación de la educación a lo largo del tiempo: una larga marcha

\section{Jean-Marie De Ketele}

\section{CpenEdition}

\section{Journals}

Édition électronique

URL : https://journals.openedition.org/ries/6893

DOI : 10.4000/ries.6893

ISSN : 2261-4265

Éditeur

France Education international

Édition imprimée

Date de publication : 1 décembre 2018

Pagination : $31-42$

ISBN : 978-2-85420-620-3

ISSN : $1254-4590$

Référence électronique

Jean-Marie De Ketele, «La transformation de l'éducation au fil du temps, une longue marche », Revue internationale d'éducation de Sèvres [En ligne], 79 | décembre 2018, mis en ligne le 01 décembre 2018, consulté le 24 juin 2021. URL : http://journals.openedition.org/ries/6893 ; DOI : https://doi.org/ 10.4000/ries.6893 


\title{
Figures de l'éducation dans le monde
}

\section{Introduction}

\section{La transformation de l'éducation au fil du temps, une longue marche}

\author{
Jean-Marie De Ketele \\ Université catholique de Louvain
}

Ce $79^{\mathrm{e}}$ numéro de la Revue internationale d'éducation de Sèvres s'intéresse aux grandes figures qui, au cours des siècles, ont fait évoluer l'éducation et ont eu une influence allant au-delà de leur époque et du contexte dans lequel elles étaient apparues. Il en va des systèmes sociaux et éducatifs comme du marcheur : celui-ci peut se diriger obstinément vers un but mais aussi se tromper et revenir sur ses pas, ou rencontrer un autre marcheur, réorienter sa marche et découvrir de nouveaux horizons. La transformation de l'éducation au fil du temps est une longue marche, inséparable des contextes politiques, économiques, historiques, culturels et sociaux. Comme pour la marche, les rencontres sont susceptibles de jouer un grand rôle. Les figures évoquées ici peuvent être des personnalités qui, enracinées dans une époque et un contexte donné, se sont mises en rupture par rapport à une façon de penser et d'agir et ont cherché à transformer l'éducation. Ce peuvent être aussi, on le verra, des conceptions de l'éducation qui visent à définir des projets de société. Une nouvelle vision et de nouvelles pratiques émergent : souvent, elles auront du mal à essaimer, si ce n'est dans un cercle restreint de disciples; presque toujours, elles trouveront un accueil dans d'autres lieux et auront même une audience internationale très forte ; et elles finiront, dans des délais plus ou moins longs, selon les cas, par transformer le contexte d'origine et les systèmes dans lesquels elles se sont disséminées.

De telles personnalités entraînent dans leur marche d'autres personnes ; un mouvement se crée, porté par des hommes et des femmes plus ou moins mis en lumière ou par des travailleurs de l'ombre, et se ramifie éventuellement. Si, faute de place, nous n'avons pu consacrer dans ce numéro un espace spécifique à toutes les figures ou à tous les mouvements qui l'auraient mérité, nous nous sommes attachés à présenter certaines personnalités de l'éducation devenues incontournables depuis longtemps et partout, et d'autres moins connues, notamment du grand public. Qui contesterait, par exemple, la présence de Confucius dans ce numéro ? Ou ne serait heureux de découvrir, peut-être, l'importance d'une figure telle que Grundtvig, ce Danois qui a tant influencé les pays scandinaves, actuellement à l'honneur dans les enquêtes internationales ? Qui, à travers les mouvements étudiés, ne prendra pas plaisir à retrouver le nom de personnalités engagées, qui auraient certainement 
mérité un article spécifique ? De Dewey à Montessori, d'Averroès et Ibn Khaldûn à Vygotski ou Piaget, de Freire à Freud, Abreu, Condorcet, Rousseau ou Ferry, de la Paideia à l'évolution des conceptions de l'éducation en Afrique, un large éventail d'époques et de contextes est proposé au lecteur.

Les auteurs sollicités se sont attachés, généreusement, à présenter au mieux les figures ou les mouvements étudiés. Ils les situent avec précision dans le contexte de leur émergence et font apparaître clairement la dimension de rêve ou d'utopie qu'ils véhiculaient. Il nous reste à articuler, en guise d'introduction, les cheminements qui les réunissent, en sollicitant éventuellement les liens qui ont été tissés au fil des siècles avec d'autres figures ou mouvements qui n'ont pas fait l'objet de contributions. Ce faisant, nous répondons à l'objectif qui a présidé à l'élaboration de ce numéro : tenter de mettre en évidence la chaîne des événements, des idées et des ruptures qui laissent entrevoir le cheminement futur de l'éducation.

Pourrons-nous, en ces temps actuels de grandes mutations sociétales, voir ce futur comme une recomposition du passé face à de nouvelles opportunités offertes par le présent ? La question n'est pas anodine pour la Revue internationale d'éducation, qui prépare un colloque international en juin 2019 sur les conditions de réussite des réformes en éducation.

\section{DES FIGURES AU FONDEMENT DES CIVILISATIONS...}

À travers quelques-unes des grandes figures étudiées (personnalités et mouvements), nous pouvons repérer quatre grands chemins, au sein de quatre civilisations, qui ont traversé les siècles jusqu'à nos jours.

La première figure que nous évoquerons remonte bien avant notre ère, puisque Confucius est né en Chine, en 551 av. J.-C. Son projet social et éducatif pour créer une société harmonieuse se trouve au fondement de la «culture sinisée ", dont les valeurs ne sont pas étrangères aux performances actuelles remarquables de certains pays d'Asie, comme l'a mis en évidence le colloque international «L'éducation en Asie : quels enjeux mondiaux? (12-14 juin 2014), objet du n 68 de la présente revue. Cette transmission a pu se faire grâce à l'existence de disciples, voire de dissidents, qui ont interprété ou adapté son œuvre en fonction des contextes qui étaient les leurs. Le fait qu'ils y aient intégré des éléments issus du bouddhisme et du taoïsme n'est pas étranger à la pénétration du confucianisme dans de nombreuses régions d'Asie, pénétration facilitée sans doute par le commerce chinois le long de certaines voies marchandes.

De l'autre côté de la planète, la civilisation grecque a inventé le concept de Paideia, complexe et difficile à traduire. Soucieux de former une élite capable de bien gérer la "Cité », les philosophes, dont Aristote et Platon, accordaient beaucoup d'importance au fait d'enseigner à celle-ci une vaste culture, sur la base des disciplines connues de l'époque, pour "é-lever l'enfant » (lever l'enfant au-delà pour atteindre l'excellence, selon Platon). La Paideia est donc à la fois éducation et culture d'une élite. Cette conception de l'éducation va se transmettre dans tout le monde latin, puis chrétien, vivre des transformations à la Renaissance et au siècle des Lumières (notamment en s'appuyant sur les écrits du monde arabo-musulman), s'étendre 
au-delà de l'Europe au moment de la colonisation de l'Amérique latine puis de l'émigration vers l'Amérique du Nord. On peut parler de civilisation occidentale, même si elle est composée de sous-cultures différenciées (dont notamment l'anglo-saxonne, qui prendra de plus en plus d'importance au fil du temps).

La civilisation arabo-musulmane, quant à elle, va se développer autour des sciences religieuses, qui seront le socle exclusif de l'éducation ; elle va rapidement s'étendre au Moyen-Orient, en Asie occidentale, au Maghreb et en Andalousie. Deux figures importantes, Averroès et Ibn Khaldûn, vont tenter de sortir l'éducation de cet enfermement en distinguant les sciences religieuses, non démontrables, et les sciences profanes reposant sur la "certitude " (l'observation et la démonstration). Bien que, pendant de nombreuses années et dans de nombreux lieux, leurs écrits aient eu peu d'influence sur le monde musulman, ils vont être diffusés en grande partie par les Juifs andalous et leurs successeurs, par le biais des traductions en hébreu, puis dans d'autres langues. Les intellectuels des siècles suivants trouveront chez Averroès et Ibn Khaldûn l'amorce du concept de laïcité et l'embryon de la didactique moderne, à travers leurs propositions d'organisation de l'enseignement.

Bien avant l'émergence de ces trois civilisations existaient des civilisations " originelles », dans lesquelles l'éducation se faisait essentiellement par deux vecteurs : la proximité avec la nature, ressource de la vie qu'il était nécessaire de bien connaître ; la tribu ou la communauté d'appartenance, appelée souvent " la famille élargie ", dont les anciens transmettaient la sagesse (au sens étymologique du terme, sophia désignant la connaissance pour vivre en société). On en trouve encore des traces profondes en Afrique noire et dans certaines régions rurales isolées de plusieurs continents. Si ces aspects de cette éducation ne sont pas abordés explicitement dans l'article de Masengesho Kamuzinzi, celui-ci s'attache à retracer un parcours qui va de la colonisation occidentale au passage de la communauté proche à la " communauté classe », puis plus récemment, à l'évolution de la communauté classe à des systèmes éducatifs de plus en plus "globalisés » (influencés par une pensée unique, malgré la pluralité des Afriques), à travers le rôle joué par les organismes internationaux de financement, notamment. Cette histoire n'ayant guère été marquée par des figures incontournables reconnues universellement, on peut se demander si la volonté de certains intellectuels africains actuels de réécrire leur histoire parviendra à produire à terme une vision renouvelée de l'éducation. Afrique, continent de demain, dit-on. L'éducation, au service de qui et de quoi ?

Les quatre grandes civilisations que nous venons d'évoquer couvrent toutes les régions du monde, à l'exception sans doute de la civilisation hindoue, dont deux grandes figures ont émergé tardivement, à la transition des $\mathrm{XIX}^{\mathrm{e}}$ et $\mathrm{xx}^{\mathrm{e}}$ siècles : le grand écrivain Rabindranath Tagore et le Mahatma Gandhi, qui auraient pu trouver une juste place dans ce numéro. Bien avant eux, 1500 ans avant notre ère, l'éducation s'inscrivait dans la tradition religieuse sur la base du manuscrit, le veda. Limitée à la classe intellectuelle des Brahmanes, l'éducation se faisait autour d'un précepteur ( $g u r u$ ), dans des lieux proches de la nature, ou plus tard au sein des monastères bouddhistes. La colonisation britannique a profondément changé l'éducation, en important en Inde le système britannique et en créant des écoles mais aussi des universités à Bombay, à Calcutta et à Madras, sur le modèle de l'Université de Londres. Malgré les tentatives de Tagore et de Gandhi, le système 
britannique a perduré et il est même à la base de la formation d'un grand nombre de scientifiques indiens (particulièrement en mathématiques et en informatique), que d'autres pays s'arrachent, comme les États-Unis. La tension entre tradition et modernité, que nous rencontrons dans bien d'autres régions du monde, n'en reste pas moins repérable.

\section{... ET DES FIGURES AU FONDEMENT DE L'ÉDUCATION CONTEMPORAINE}

L'éducation contemporaine a été façonnée progressivement au cours des siècles et il faut bien constater que c'est d'abord l'Europe occidentale, puis l'Amérique du Nord (effet de l'émigration) et du Sud (réaction à la colonisation) qui ont été à la base de cette dynamique. Peut-être en sera-t-il autrement demain, avec la montée en puissance des pays asiatiques.

On peut sans doute attribuer les premières amorces de l'éducation contemporaine à une lignée de grandes figures ayant soit préfiguré la Révolution française (Rousseau), soit vécu celle-ci (Condorcet), soit mis en œuvre concrètement ses idées en matière d'éducation (Ferry). N'oublions pas que la Paideia à la base de la civilisation occidentale se tournait vers la formation d'une élite. Rousseau sera l'un des précurseurs de la Révolution française avec son Discours sur l'inégalité. En 1791, Condorcet jettera les bases de l'idéal d'égalité de la République naissante dans Mémoires de l'instruction publique. Pour rendre effective l'instruction pour tous, Ferry, en homme politique pragmatique, organisera l'instruction primaire laïque, gratuite et obligatoire. Initiée au XVI ${ }^{\mathrm{e}}$ siècle lors de la Réforme et de la Contre-Réforme, la " forme scolaire », une certaine manière d'organiser l'école et de transmettre le savoir et le savoir-faire, est adoptée par l'État français. On la retrouvera jusqu'à nos jours dans la majorité des pays, à tous les niveaux de la scolarité, au collège et au lycée, et même dans l'enseignement supérieur, sous l'influence de Humboldt en Allemagne et bien au-delà. Cependant, l'idéal d'égalité prôné par les fondateurs de la République sera loin d'être atteint ; des sociologues, comme Bourdieu, dénonceront le déterminisme social renforcé par l'école. La recherche de l'équité deviendra un enjeu de plus en plus aigu des systèmes éducatifs contemporains, y compris pour l'OCDE, qui est pourtant, comme son nom l'indique, un organisme de développement économique.

$\mathrm{Au}$ vingtième siècle, de nombreuses figures vont remettre en question certains aspects de la forme scolaire, principalement son organisation et le mode de transmission du savoir et du savoir-faire. Nous en avons retenu quatre, dont les conceptions ont entraîné la création de mouvements pédagogiques importants : Piaget, le Genevois fondateur du mouvement d'éducation nouvelle et du constructivisme ; Vygotski, le Russe inspirateur du socio-constructivisme qui fera évoluer le mouvement d'éducation nouvelle ; Dewey, l'Américain défenseur d'un apprentissage par l'expérience et comme processus social et démocratique ; Freud, l'Autrichien qui, en mettant en évidence le rôle de l'inconscient, va amener les psychanalystes à attirer l'attention sur l'importance de la relation éducative et de ses dérives.

Autour de ces grandes figures, on assiste un peu partout à un bouillonnement d'idées et d'initiatives, qui auraient également mérité une analyse que nous n'avons pu proposer, faute de place. Citons, à titre d'exemples, parmi beaucoup 
d'autres : en Suisse, Ferrière et son école à la campagne ; en France, Freinet et le tâtonnement expérimental, mais aussi Oury et la pédagogie institutionnelle inspirée de la psychanalyse ; en Écosse, Neil et l'autogestion des enfants de Summerhill ; en Pologne, Korczak et son orphelinat organisé en république des enfants ; en Espagne, Ferrer et la pédagogie libertaire de l'Escuela moderna ; en Allemagne, Kerschensteiner et les écoles du travail, mais aussi Steiner et ses écoles basées sur sa doctrine " anthroposophique »; aux États-Unis, Rogers et le développement de la personne... Si ces initiatives ont pour caractéristique commune de remettre en question certains aspects de la forme scolaire, elles n'ont pas réussi à véritablement pénétrer en profondeur les systèmes éducatifs nationaux, malgré un accueil attentif du milieu de la recherche et, sur le plan des idées du moins, des institutions de formation des enseignants.

Par ailleurs, un certain nombre de figures ont élargi la notion d'éducation, trop souvent réduite à l'enseignement obligatoire et à la forme scolaire. Cet élargissement s'est fait en amont, en aval et à côté de l'école.

En amont, on trouve l'Italienne Maria Montessori, femme engagée contre le fascisme, qui va montrer l'importance de l'éducation des jeunes enfants et proposer à cet effet une méthode rigoureuse, qui s'écarte autant du gardiennage que de la forme scolaire classique.

En aval, deux grandes figures vont révolutionner l'éducation des adultes, champ négligé jusqu'alors. D’abord, le Danois Grundtvig contribue, au XIx ${ }^{\mathrm{e}}$ siècle, à fonder l'éducation populaire dans les pays scandinaves et à populariser le concept d'éducation tout au long de la vie, qui sera repris ensuite par des organismes internationaux, et notamment la Commission européenne, qui a donné son nom à un important programme d'éducation tout au long de la vie. Ensuite, le Brésilien Paulo Freire, frappé par l'importance de l'analphabétisme et de ses conséquences néfastes, va mettre au point une pédagogie destinée aux adultes, afin non seulement d'alphabétiser ces derniers, mais aussi et surtout de les libérer des oppressions politiques, sociales et culturelles qu'ils subissent. Cette pédagogie de la libération va largement s'étendre au plan international et va même inspirer la Conférence mondiale de l'Unesco sur l'enseignement supérieur, en reprenant le concept d'éducation comme bien public, cher à Paulo Freire.

À côté de l'institution scolaire et en s'écartant de la forme scolaire, le Venezuelien José Antonio Abreu, qui vient de nous quitter en mars 2018, est parvenu en quelques années à développer un mouvement mondial, El Sistema, qui donne à tous, jeunes et adultes, accès à l'éducation musicale. Celle-ci, comme plus largement l'éducation artistique, n'est pas au centre des préoccupations de l'école, alors que, nous l'explique Anis Barnat, " la pratique collective de la musique est un droit fondamental, un modèle humaniste d'intégration et d'organisations sociales ». Des orchestres El Sistema, mélangeant tous les âges et toutes les conditions sociales ou ethniques, ont fleuri un peu partout dans le monde.

Grundtvig, Freire et Abreu nous rappellent que l'éducation contemporaine et à venir ne peut se limiter à l'intérieur des murs de l'école ; elle doit être pensée dans tous les champs qui caractérisent la vie des personnes, à tous les âges de la vie. Et il est curieux de constater que les pratiques utilisées hors du champ de l'école se sont en général plutôt inspirées des conceptions éducatives des grandes figures qui n'étaient pas parvenues à réformer l'institution scolaire. 


\section{ENRACINEMENT ET RUPTURE, AU FONDEMENT DES VISIONS NOUVELLES}

Chacune des figures étudiées dans ce numéro s'inscrit dans une période précise de l'histoire et dans un contexte politique, social et culturel. Contrairement à leurs contemporains, ces figures sont sorties de l'anonymat en se mettant en rupture par rapport aux visions de l'époque et en formulant des propositions inédites qui, très souvent, ont été mieux reçues à l'extérieur, du moins dans un premier temps.

Si l'on observe les grandes figures présentées dans ce numéro, on peut distinguer trois grands types d'enracinement et de rupture : d'une part de nature politique et culturelle, sous l'angle de la gouvernance de la société ; ensuite de nature scientifique, dans une période de développement des sciences humaines; enfin de nature sociale, en lien avec les laissés-pour-compte de la société et de l'éducation.

Dans la première catégorie, on trouve d'abord des personnalités confrontées à l'effritement d'une situation politique et sociale. C'est le cas de Confucius, dont la nostalgie est double : nostalgie de la dynastie précédente, dont la stabilité politique reposait sur l'importance attribuée aux " rites "; nostalgie de sa région de naissance, réputée celle des " hommes de bien ». Ces deux concepts seront à la base de son projet social et éducatif. Il est curieux de constater le parallélisme avec les philosophes grecs, particulièrement Aristote et Platon, dont le projet éducatif est de former des " citoyens » capables de gérer la Cité, dans un contexte où les Cités sont souvent amenées à se défendre. Si Averroès a une carrière d'enseignant dans toutes les grandes cités intellectuelles du monde arabo-musulman de son époque, Ibn Khaldûn assiste au contraire, impuissant, à l'effritement de ce monde. Tous deux cependant, au contact de la pensée des grands philosophes de l'Antiquité, vont tenter de se dégager de la domination des sciences religieuses et théologiques pour montrer l'importance, dans la formation des jeunes, des sciences profanes, fondées sur des critères scientifiques. C'est encore le cas de la lignée de figures telles que Rousseau et Condorcet, confrontées à l'effritement du pouvoir des nobles et du clergé et à la montée en puissance de la révolte du peuple face aux inégalités ressenties et aux difficultés pour vivre.

Dans la deuxième catégorie, c'est le contexte scientifique de l'époque, et non le contexte politique, qui est à la base des nouvelles visions de l'éducation. Au XVIII $^{\mathrm{e}}$ siècle, le projet de l'Encyclopédie témoignait de l'essor des sciences et des techniques. La fin du XIX $x^{e}$ siècle et la première moitié $\mathrm{du} \mathrm{Xx}^{\mathrm{e}}$ siècle seront marquées par l'avènement des sciences dures et, dans la foulée, des sciences humaines. Dans ce contexte de bouillonnement scientifique, les nouvelles visions se créeront en rupture par rapport à certaines conceptions dominantes des sciences humaines de l'époque et seront génératrices de disciplines nouvelles ayant une incidence sur les projets éducatifs. Ainsi, Piaget ne pourra se satisfaire de la psycho-physique, créée en Allemagne par Fechner et Wundt, ou du behaviorisme qu'il découvrira lors de son voyage aux ÉtatsUnis ; il sera donc à l'origine de la psychologie du développement, avec sa théorie des stades et du constructivisme ( «la connaissance se construit par l'enfant par son action sur l'environnement ») ; ses découvertes auront des incidences majeures sur l'éducation et seront à la base du mouvement d'éducation nouvelle, qu'il fondera. 
De son côté, Vygotski crée la psychologie russe, en accord avec ses idées militantes du collectivisme communiste mais en dépassant le constructivisme de Piaget, un auteur qu'il a lu : à un certain moment de l'apprentissage (d'où le concept de "zone proximale de développement »), l'enfant a besoin de l'adulte et du langage pour progresser ; l'apprentissage est un processus social et culturel. Le socio-constructivisme allait pénétrer le mouvement d'éducation nouvelle et le faire évoluer, grâce aux nombreuses figures évoquées précédemment.

De l'autre côté de l'Atlantique, dans un contexte marqué par l'empirisme de Locke, John Dewey se démarque du behaviorisme de son époque et de Benjamin Bloom, fondateur de la " pédagogie de maîtrise » et précurseur du " pilotage par les résultats ». Ce ne sont ni le conditionnement opérant ni le recours à des objectifs bien définis et opérationnalisés qui caractérisent l'apprentissage, selon Dewey, mais le recours à l'expérience, le fait d'offrir à chaque élève l'opportunité de mettre en ouvre ses « capacités distinctives » et de les mettre au service du groupe. De cette façon, l'éducation est au service de la démocratie (concept-clé de son œuvre philosophique et de son projet éducatif).

Freud, médecin autrichien, se met en rupture de la psychiatrie traditionnelle et de la psycho-physique allemande. Influencé par les travaux de Charcot sur l'hypnose et l'hystérie, il met en évidence le rôle de l'inconscient chez l'être humain, notamment dans la relation du médecin et de son patient ; il crée une nouvelle science. Ses travaux seront repris par les psychanalystes qui étudieront la relation éducative, et par le mouvement de la pédagogie institutionnelle. Ici encore, l'approche psychanalytique restera essentiellement cantonnée à la thérapie et ne pénétrera guère l'institution scolaire, à l'exception de rares écoles privées (par exemple, celles qui se réclament de Françoise Dolto).

Dans la troisième catégorie, certains vont se préoccuper d'un public de laissés-pour-compte du champ de l'éducation et vont, nous l'avons dit, élargir le champ de l'éducation. Médecin elle-même, Maria Montessori participe au mouvement des médecins de son époque, qui va révolutionner la façon de s'occuper des orphelins et des enfants considérés comme sortant de la norme sociale. Elle s'inspire de ces travaux pour créer la Casa dei bambini à Rome et expérimenter une méthode d'éducation des jeunes enfants. Elle prend ainsi le relais de Froebel qui, au XIX siècle, avait créé des " jardins d'enfants ». Ces deux auteurs sont à la base de l'institutionnalisation de l'éducation dite froebélienne ou encore préscolaire. Les travaux des chercheurs en éducation montreront ultérieurement toute l'importance des apprentissages conduits pendant cette période du développement de l'enfant.

À l'autre bout de la chaîne éducative, N. F. S. Grundtvig et Paulo Freire, en s'occupant des adultes laissés-pour-compte, vont être à l'origine des mouvements d'éducation populaire, de la pédagogie des adultes et de l'éducation tout au long de la vie. Enraciné dans la tradition protestante de lecture personnelle de la Bible, Grundtvig va mettre l'accent sur l'alphabétisation par l'apprentissage de la lecture, dans un souci d'une éducation pour tous qui continue à caractériser les systèmes éducatifs scandinaves. Paulo Freire, quant à lui, confronté à un contexte brésilien dans lequel une large partie de la population est opprimée sous le joug de la colonisation, va, au-delà d’une alphabétisation nécessaire, créer une " pédagogie de la libéra- 
tion », dans le but de voir les opprimés prendre eux-mêmes en charge leur place dans la société. Le mouvement tiers-mondiste s'inspirera largement de cette pédagogie de la libération.

À côté de la chaîne éducative institutionnalisée, Abreu, enfin, va se préoccuper d'une autre catégorie de laissés-pour-compte : ceux qui n'ont pas la possibilité, faute de moyens, de participer à la culture, réservée à une élite favorisée. Si l'action d'El Sistema concerne la musique, la culture artistique et la pratique des arts, de façon plus large, ne participent-elles pas à la construction identitaire et sociale de la personne?

\section{LA NÉCESSAIRE ALLIANCE ENTRE LA TRADITION ET LA MODERNITÉ POUR RÉPONDRE AUX DÉFIS ACTUELS}

Nous avons tenté jusqu'ici d'identifier les cheminements de l'éducation à travers les siècles. La marche de l'éducation se poursuit inexorablement. Trois questions se posent alors : quels sont les concepts-clés apportés par la tradition et qui continueront à traverser l'éducation en marche ? Quelles sont les mutations actuelles véhiculées par la modernité et qui offrent des opportunités de rupture pour de nouvelles visions de l'éducation ? En quoi la tradition et la modernité s'allieront-elles pour faire système et tenter ainsi de répondre aux défis posés par ces mutations actuelles?

\section{Des concepts-clés issus de la tradition}

Plusieurs concepts-clés (et les questions qu’ils véhiculent) traversent le cheminement des visions de l'éducation à travers les siècles ; pour reprendre la pensée d'Aristote sur le sujet, ce sont en quelque sorte des universaux qui se recomposent et se particularisent, selon les périodes et les contextes.

Un premier concept-clé apporté par la tradition est lié à l'un des buts fondamentaux de l'éducation, tel qu'il émerge des deux racines étymologiques du terme : e-ducare (nourrir, élever) et $e$-ducere (faire sortir de, lever plus haut). C'est bien ce que poursuit Confucius, qui cherche à former des « hommes de bien »; ou ce que visent la Veda et Tagore, l'harmonie et la perfection, présentes dans la nature ou encore les philosophes grecs avec la Paidea, l'excellence. "Quel développement de la personne? » est une question qui traverse toute l'histoire de l'éducation.

Un deuxième concept-clé se réfère à un autre but de l'éducation, celui de servir la société : créer une société harmonieuse, dit Confucius ; former des citoyens capables de bien servir la Cité, pour les philosophes grecs ; créer une nation démocratique, où chaque élève développe au sein de la classe ses capacités distinctives et les met au service du groupe, selon Dewey. "Quelle éducation pour servir quelle société à construire? » est une autre interrogation fondamentale. 
Un troisième concept-clé porte sur l'instruction souhaitée pour élever l'enfant et ainsi servir la société : les sciences profanes, qui permettent le développement de la capacité à argumenter sur la base de critères scientifiques, diront Averroès et Ibn Khaldûn ; l'instruction publique gérée par la République, estimeront Condorcet et Ferry ; l'action de l'enfant sur l'environnement, pour Piaget ; l'action de l'adulte et du langage, pour Vygotski ; la présentation au jeune enfant d'un matériel adapté à son âge et à ses besoins, pour Montessori ; une relation éducative et affective saine, pour l'approche psychanalytique; la pratique collective de la musique, pour Abreu. Cette question « quelle instruction ? » pose la question de la pertinence, c'està-dire du rapport entre l'apprentissage réellement effectué (instruction assimilée) dans un contexte donné et l'apprentissage qu'il faudrait faire (instruction à assimiler) dans ce contexte.

Un quatrième concept-clé cerne l'audience et le lieu de l'éducation : au fil des siècles, on prend conscience qu'il ne faut pas réduire l'éducation à un type de public ou à un âge donné ou dans un lieu donné (Confucius, la Paideia), mais à tous (Condorcet et Ferry), à tous les âges et tout au long de la vie (Montessori, Grundtvig et Freire), dans tous les lieux (de la communauté comme dans l'éducation originelle africaine, à l'école ou à côté de l'école, comme chez Abreu ou dans le champ du travail étudié actuellement par la didactique professionnelle). L'histoire de l'éducation construit une trajectoire, depuis une éducation de qualité qui prône l'excellence et l'élitisme à une éducation de qualité pour tous. D'où la question : " comment concilier excellence et éducation pour tous?».

Deux nouveaux concepts-clés sont induits à la fois par cet élargissement et par un mouvement peu ou guère abordé dans ce numéro. Si nous avons évoqué le bouillonnement scientifique et l'avènement de certaines sciences humaines aux $\mathrm{XIX}^{\mathrm{e}}$ et $\mathrm{XX}^{\mathrm{e}}$ siècles, il faut rappeler que ceci est dû au développement extraordinaire des sciences dites exactes. Si des sciences humaines, comme la psychanalyse, se sont nettement démarquées du paradigme expérimental et quantitatif, d'autres ont tenté de l'appliquer à l'étude de l'humain et du social. C'est ce qu'ont tenté de faire le behaviorisme et la pédagogie de maîtrise de Bloom, laquelle a eu un impact dans la plupart des régions du monde, avant la montée en puissance de l'approche par compétences. Mais il faut souligner aussi que ce mouvement a été prolongé par la naissance de sciences telles que la docimologie, la psychométrie, l'économétrie, l'édumétrie, etc., qui ont contribué à instaurer (voire à imposer, souvent) une culture de l'évaluation, avec l'avènement des évaluations internationales et nationales et un mode de gouvernance basé sur la preuve. Deux concepts sont alors développés, qui ont pris une place de plus en plus importante au cours des dernières décennies : celui d'efficacité, comme rapport entre les effets réellement observés ou évalués ou mesurés et les effets attendus par les acteurs concernés ; et celui d'équité, comme distribution égale des bénéfices de l'éducation à travers les différents groupes de population, sans discrimination de genre, de classe sociale, d'origine, de territoire... Si un consensus s'établit sur la nécessité d'avoir une éducation efficace et équitable, doit-elle être toujours efficiente (rapport entre les effets observés et les ressources investies) ? Aujourd'hui, la question centrale est la suivante : " comment assurer une éducation à la fois pertinente, efficace et équitable? ». 


\section{La modernité et son lot de mutations}

La modernité est une notion relative. À cette étape de notre raisonnement, il nous importe de nous interroger sur les mutations et opportunités de rupture qui caractérisent notre décennie. Elles sont très nombreuses et en fortes interactions. Nous les caractériserons par quatre tendances très nettes : (1) l'accélération du développement des technologies ; (2) le développement de disciplines aux enjeux sociétaux et éthiques importants ; (3) la montée en puissance de la globalisation ; (4) les phénomènes de polarisation et leurs conséquences.

Une première catégorie de mutations a trait à l'accélération du développement des technologies. Chaque personne, y compris les jeunes enfants, dispose d'outils technologiques toujours plus puissants, comme les smartphones, qui donnent accès à des bases de données de plus en plus exhaustives et à des moteurs de recherche de plus en plus rapides. Le maître de l'information n'est plus le savant dans nos sociétés ni l'enseignant dans nos écoles. Parallèlement se développent les technologies de la mobilité physique et virtuelle : les opportunités de voyager dans d'autres pays et d'autres cultures se sont multipliées, comme en témoignent des initiatives telles que le programme Erasmus, parmi d'autres ; mais les opportunités de mobilité virtuelle ne sont pas en reste, grâce à une multiplicité d'applications comme Skype et de réseaux sociaux, qui sont utilisés dans divers champs de l'activité humaine. Il n'y a pas longtemps, de telles technologies étaient impensables ; elles deviennent indispensables maintenant et ceci ne va pas sans poser de graves questions, notamment en ce qui concerne la validité des informations (les «fake news») et les usages des applications (on pense au harcèlement au sein des établissements scolaires ou à leur utilisation à des fins de terrorisme).

Le développement de certaines disciplines de pointe apporte des évolutions offrant des perspectives nouvelles dans les différents champs de l'activité humaine. C'est ainsi le cas des sciences biologiques pouvant agir sur le génome humain ; ou des neurosciences qui montrent le lien étroit entre cognition et émotion et le rôle des neurones « miroir »; ou encore de l'intelligence artificielle qui, dopée par la puissance de stockage et de traitement de l'information et de traitement, remplace de plus en plus l'être humain. D’énormes enjeux éthiques sont posés.

En conséquence de ces nombreuses mutations se développe la « globalisation ", ce processus d'émergence d'un système-monde qui tend à s'imposer comme un modèle unique, universellement reconnu, transnational et transculturel, affectant tous les domaines de l'activité humaine (y compris l'éducation), s'étendant à l'ensemble de l'espace terrestre et nécessitant une gestion à l'échelle du globe. Partout dans le monde, on retrouve les mêmes produits de consommation, y compris culturels. Les interdépendances se multiplient dans la plupart des secteurs de l'activité humaine, sans en oublier les conséquences, comme le dérèglement climatique. Les gouvernances nationales laissent de plus en plus la place aux gouvernances supranationales (OCDE, Banque mondiale, Fonds monétaire international, ONU, Unesco, Unicef, Union européenne...). Des inquiétudes se manifestent devant l'imposition sournoise d'une culture et d'une langue uniques et la perte des identités qui s'ensuit (ainsi, de nombreuses langues ont déjà disparu, par exemple). Les acteurs de l'édu- 
cation, tant nationaux que locaux, sont de plus en plus dépendants d'impératifs économiques et de rentabilité, sous la pression du new management et du quantifiable (les évaluations internationales et les classements, comme le montre le $\mathrm{n}^{\circ} 54 \mathrm{de}$ cette revue, consacré à cette question). Les craintes sont d'autant plus grandes qu'un organisme tel que l'Organisation mondiale du commerce tend à considérer l'éducation comme un service que le privé peut mieux rentabiliser, et à rejeter le concept de « bien public ». Ceci s'inscrit dans un contexte où, d'une part, la demande d'individualisation est très forte et où, d'autre part, des régimes dictatoriaux voudraient imposer des contenus à enseigner, dans certains pays.

Une quatrième tendance, en grande partie fruit des précédentes, est celle de la "polarisation ». Au plan des performances éducatives, l'écart entre les très bons élèves et ceux qui obtiennent de faibles résultats s'accroît plus ou moins dans tous les pays. Au plan professionnel, une hiérarchie s'établit entre métiers à haute valeur ajoutée (fruit des technologies avancées et des disciplines de pointe) et ceux qui demandent peu de qualification. Au sein des pays s'instaure une polarisation entre des territoires qui bénéficient de nombreux services de qualité (les grands centres urbains) et ceux qui se désertifient. Toutes ces polarisations se cumulent pour creuser un fossé entre certaines régions du monde, accentuant des migrations de populations vers des pays garantissant la subsistance ainsi que l'accès, pour leurs enfants, à des universités. Ces polarisations sont dangereuses et sources de rejets populaires, mais constituent des opportunités de rupture pour repenser les modèles sociaux et éducatifs ; nous avons encore en tête le rôle de Condorcet lors de la Révolution française ou encore l'action de libération entreprise par Freire, face aux polarisations de son époque. Pourquoi en serait-il autrement maintenant?

\section{Les " universaux " de la tradition pour penser de nouvelles visions}

Les mutations de la modernité actuelle (qui ne sera plus la modernité de demain) offrent des opportunités et amènent en même temps des dérives dangereuses. Les nouvelles visions qui vont marquer les décennies proches seront celles qui saisiront les premières et atténueront au mieux les secondes. Pour être « justes » (au sens de John Rawls), elles devront nécessairement s'appuyer sur les universaux mis en évidence par la tradition et donc se poser les questions suivantes : en quoi permettent-elles de développer (é-lever) la personne, grâce aux nouvelles opportunités offertes ? En quoi contribuent-elles à améliorer la gouvernance de la société ? Quelle est la pertinence du contenu et de la forme de l'apprentissage qu'elles proposent ? Parviennent-elles à allier pertinence, efficacité et équité ? Esquissons quelques pistes susceptibles d'être saisies par les grandes figures de demain.

Les data (pour utiliser un terme-clé actuellement) en accroissement permanent et les outils technologiques de plus en plus performants de traitement de celles-ci sont des opportunités pour chacun d'élever son niveau de connaissance et d'expertise. Mais s'agit-il seulement de former un homo economicus ? Certes, il servira efficacement une société du même type, grâce à l'exploitation des ressources 
offertes par les sciences les plus avancées... mais avec les conséquences que l'on sait en termes de dérèglement de l'environnement et d'accentuation des polarisations. La question «De quel homme, de quelle société avons-nous besoin ? » sera sans doute centrale dans les nouvelles visions. Elles puiseront dans la tradition, on peut l'espérer, les conceptions de l'homme de bien (Confucius), de citoyen au service de sa Cité (Aristote et Platon) mais aussi de citoyen du monde (Abreu), de chaque personne apportant dans son groupe ses capacités distinctives pour créer ainsi une société démocratique (Dewey) et ne laisser sur le bord du chemin aucun laissés-pourcompte (Grundtvig et Freire). Une grande figure sera celle qui articulera de tels éléments en système cohérent.

Les data sont multiples et non nécessairement validées ; elles sont même trafiquées pour créer des fake news à des fins discutables; les algorithmes pour les traiter sont devenus une arme de domination sociale, dit la mathématicienne américaine Cathy O’Neil ; les outils technologiques peuvent être utilisés aux dépens de personnes ciblées ; les nouvelles disciplines de pointe permettent des actions dont certaines sont éthiquement discutables. On peut donc estimer que les nouvelles visions mettront ces aspects au cœur des compétences-socles de la formation de base et développeront, aux différents âges de la vie et par des dispositifs d'apprentissage actif et participatif, une pédagogie de la libération de tout ce qui pourrait asservir des groupes de population... Les visions éducatives confucéenne et hindoue sont, de ce point de vue, particulièrement inspiratrices pour le développement de la personne ; Averroès et Ibn Khaldûn ont tenté de montrer le chemin pour ne pas enfermer l'éducation dans les sciences religieuses ; Paulo Freire est un inspirateur pour sortir des diverses polarisations évoquées. Face au trop-plein d'informations et aux tentatives de domination qu'elles véhiculent, la société et l'éducation ont besoin de maîtres qui n’asservissent pas mais libèrent par la recherche de la vérité.

Les tensions entre le global et le local, entre une culture universelle et la richesse de multiples cultures particulières, entre les gouvernances supranationales, nationales et locales, entre le quantitatif et le qualitatif, entre les disciplines dites dures et celles dites molles (et les fausses hiérarchies qui s'établissent dans les représentations sociales et dans les modes d'organisation des parcours scolaires et professionnels), entre le monde de l'école et hors des murs de celle-ci... sont autant d'aspects que, sans doute, les nouvelles visions tenteront d'articuler. Une des clés de ce travail sera de valoriser, rendre visible et reconnaître (au sens des travaux de Honneth et de Ricoeur) le travail des acteurs à leur juste place et dans un travail collectif (de nombreuses figures des mouvements éducatifs au $\mathrm{xx}^{\mathrm{e}}$ siècle ont mis en évidence l'importance du travail participatif pour lutter contre l'individualisme).

À chacun de nous, lecteurs de ce numéro, de recomposer le passé en fonction des opportunités offertes par le monde moderne actuel, pour préfigurer le devenir de l'éducation dans une société sujette aux dérives que nous avons tenté de mettre en évidence. 